\title{
ÚNG DỤNG TIN HỌC VÀO BÀI TOÁN CHUYỂ ĐỔI HỆ QUY CHIẾU BẢN ĐỒ SỐ ĐỊA HÌNH
}

\author{
ĐINH CÔNG HOÀ, HOÀNG THI! THUỶ \\ Trường Đại học Mỏ - Địa chất
}

\section{Mở đầu}

Để hiện đại hóa mạng lưới tọa độ quốc gia nhằm đáp ứng nhu cầu phát triển của đất nước trong xu thế hòa nhập vào quá trình chung của thế giới, năm 1996 Tổng cục Địa chính đã đo lưới GPS hạng "0" phủ trùm cả nước. Từ năm 1998 đến 1999 với sự tham gia của các nhà khoa học và quản lý chủ chốt của ngành đã triển khai công trình "Xây dựng hệ quy chiếu và hệ toạ độ quốc gia". Trên cơ sở đó ngày 12/07/2000 Thủ tướng chính phủ đã ra quyết định cho phép công bố và áp dụng hệ toạ độ mới của Việt Nam với tên gọi là VN2000 thay thế cho hệ toạ độ HN72. Hệ toạ độ VN2000 lấy ellipsoid thực dụng là ellipsoid WGS-84. Điểm gốc là điểm N00 trong khuôn viên Cục đo đạc và Bản đồ Việt Nam, toạ độ vuông góc phẳng theo hệ toạ độ UTM. [2]

Dữ liệu bản đồ số địa hình được thành lập trong các hệ quy chiếu khác nhau, với các kinh tuyến trục khác nhau (HN72, VN2000, WGS84). Dữ liệu bản đồ số địa hình của các đơn vị sản xuất cũng rất đa dạng, được thành lập trên cở sở các phần mềm đồ hoạ khác nhau (AutoCad, MicroStation, Mapinfo, ArcGis..). Các khuôn dạng dữ liệu đồ họa đa dạng. Vì vậy, nhu cầu của thực tế sản xuất là chuyển đổi bản đồ về cùng hệ toạ độ thống nhất để thuận lợi cho công tác quản lý và sử dụng. Đặc biệt trong thời kỳ hội nhập quốc tế, chuyển đổi dữ liệu bản đồ từ hệ tọa độ vuông góc phẳng VN2000 sang hệ quổc tê WGS84 và ngược lại là cần thiết.

Phần mềm Geosoft đã có các chức năng tự động chuyển đổi bản đồ về hệ toạ độ mong muốn từ dữ liệu ở các hệ quy chiếu, kinh tuyến trục và khuôn dạng dữ liệu khác nhau, đảm bảo bảo toàn mối quan hệ hình học của các đối tượng không gian và không làm thay đổi các thuộc tính của đối tượng, kết quả tính chính xác, trực quan thể hiện trên phần mềm đồ họa.

\section{Công thức tính chuyển đổi tọa độ bản đồ số địa hình}

a) Công thức tính chuyển từ tọa độ trắc địa $(B, L)$ sang tọa độ vuông góc phẳng $(x, y)$

$$
\begin{aligned}
& (x-X)=\frac{N}{2 \rho^{\prime \prime 2}} \sin B \cos B 1^{\prime \prime 2}+\frac{N}{24 \rho^{\prime 4}} \sin B \cos ^{3} B\left(5-t^{2}+9 \eta^{2}+4 \eta^{2}\right) 1^{1{ }^{4}}+\frac{N}{720 \rho^{\prime \prime 6}} \sin B \cos ^{5} B\left(61-58 t^{2}+t^{4}\right) 1^{11^{6}} \\
& y=\frac{N}{\rho^{\prime \prime}} \cos B 1^{\prime \prime}+\frac{N}{6 \rho^{\prime 3}} \cos ^{3} B\left(1-t^{2}+\eta^{2}\right) 1^{13}+\frac{N}{120 \rho^{\prime \prime}} \cos ^{5} B\left(5-18 t^{2}+t^{4}+14 \eta^{2}-58 t^{2} \eta^{2}\right) 1^{15}
\end{aligned}
$$

b) Công thức tính chuyển từ tọa độ vuông góc phẳng $(x, y)$ sang tọa độ trắc địa $(B, L)$

$$
\begin{aligned}
& B_{1}-B=\frac{\rho^{\prime \prime} t_{1}}{2 M_{1} N_{1}} y^{2}-\frac{\rho^{\prime \prime} t_{1}}{2 M_{1} N_{1}^{3}}\left(5+3 t_{1}^{2}+\eta_{1}^{2}-9 t_{1}^{2} \eta_{1}^{2}\right) y^{4}+\frac{\rho^{\prime \prime} t_{1}}{720 M_{1} N_{1}^{5}}\left(61+90 t_{1}^{2}+45 t_{1}^{2}\right) y^{6} \\
& l=\frac{\rho^{\prime \prime}}{N_{1} \cos B_{1}} y-\frac{\rho^{\prime \prime}}{6 N_{1}^{3} \cos B_{1}}\left(1+2 t_{1}^{2}+\eta_{1}^{2}\right) y^{3}+\frac{\rho^{\prime \prime}}{120 N_{1}^{5} \cos B_{1}}\left(5+28 t_{1}^{2}+24 t_{1}^{4}+6 \eta_{1}^{2}+8 t_{1}^{2} \eta_{1}^{2}\right) y^{5}
\end{aligned}
$$


Trong các công thức trên, $\mathrm{X}$ là chiều dài cung kinh tuyến, được xác định dựa vào độ vĩ $\mathrm{B}$ đã biết; $\mathrm{N}$ là bán kính cong vòng thẳng đứng thứ nhất; hệ số $\mathrm{I}, \mathrm{M}, \mathrm{N}, \mathrm{t}, \mathrm{h}$ được tính như sau:

$\mathrm{I}=\mathrm{L}-\mathrm{L}_{0}$, ( $\mathrm{L}_{0}$ là kinh tuyến trục); $\mathrm{t}=\operatorname{tg} \mathrm{B} ; \mathrm{t}_{1}=\operatorname{tg} \mathrm{B}_{1} ; \mathrm{h}^{2}=\mathrm{e}^{\prime 2} \cos ^{2} \mathrm{~B}$;

$M=\frac{a\left(1-e^{2}\right)}{\left(1-e^{2} \sin ^{2} B\right)^{3 / 2}} ; N=\frac{a}{\left(1-e^{2} \sin ^{2} B\right)^{1 / 2}}$

e là độ lệch tâm thứ nhất, e' là độ lệch tâm thứ hai; a là bán trục lớn của Elipxoid.

c) Tính chuyển toạ độ $X, Y, Z$ trong Hệ WGS-84 quốc tế sang $X_{1}, Y_{1}, Z_{1} H$ ệ VN-2000

$$
\begin{aligned}
& \mathrm{X}_{1}=\Delta \mathrm{X}_{0}+\mathrm{k} \cdot\left(\mathrm{X}-\omega_{0} \cdot \mathrm{Y}+\psi_{0} \cdot \mathrm{Z}\right) \\
& \mathrm{Y}_{1}=\Delta \mathrm{Y}_{0}+\mathrm{k} \cdot\left(\omega_{0} \cdot \mathrm{X}+\mathrm{Y}-\varepsilon_{0} \cdot \mathrm{Z}\right) \\
& \mathrm{Z}_{1}=\Delta \mathrm{Z}_{0}+\mathrm{k} \cdot\left(-\psi_{0} \cdot \mathrm{X}+\varepsilon_{0} \cdot \mathrm{Y}+\mathrm{Z}\right)
\end{aligned}
$$

Trong đó:

k là tỷ lệ biến dạng chiều dài của Hệ WGS-84 quốc tế so với Hệ VN-2000,

$\omega_{0}, \Psi_{0}, \varepsilon_{0}$ là góc quay Ơ-le của trục toạ độ Hệ WGS-84 quốc tế so với Hệ VN-2000,

$\Delta \mathrm{X}_{0}, \Delta \mathrm{Y}_{0}, \Delta \mathrm{Z}_{0}$ là toạ độ tâm của Hệ WGS-84 quốc tế trong Hệ VN-2000.

d). Tính chuyển toạ độ $B, L, H$ sang tọa độ vuông góc không gian $X, Y, Z$

Chuyển đổi từ hệ tọa độ trắc địa $\mathrm{B}, \mathrm{L}, \mathrm{H}$ sang tọa độ vuông góc không gian $\mathrm{X}, \mathrm{Y}, \mathrm{Z}$ của cùng một điểm (trong một hệ thống tọa độ) ta sử dụng công thức sau:

$$
\begin{aligned}
& X=(N+H) \cos B \cdot \cos L \\
& Y=(N+H) \cos B \cdot \sin L \\
& Z=\left[N\left(1-e^{2}\right)+H\right) \sin B
\end{aligned}
$$

Quá trình chuyển đổi $(X, Y, Z)$ sang $(B, L, H)$ được thực hiện theo các công thức:

$$
\operatorname{tg} L=\frac{Y}{X} ; \operatorname{tg} B=\frac{Z+N e^{2} \cdot \sin B}{R} ; \quad \mathrm{H}=\mathrm{Z} / \sin \mathrm{B}-\mathrm{N}\left(1-\mathrm{e}^{2}\right) ; \quad \mathrm{R}=\left(\mathrm{X}^{2}+\mathrm{Y}^{2}\right)^{1 / 2}
$$

Việc xác định $B$ được tiến hành theo phương pháp nhích dần.

\section{Chuyển đổi Hệ quy chiếu bản đồ số địa hình}

Từ tệp dữ liệu đồ hoạ của bản đồ số, bằng việc phân tích khuôn dạng dữ liệu [1], chúng ta xác định được giá trị toạ độ của các đối tượng đồ hoạ, từ đó chuyển đổi toạ độ của chúng sang hệ toạ độ mới. Để chuyển đổi bản đồ qua các múi chiếu có kinh tuyến trục khác nhau, căn cứ toạ độ $x, y$ theo công thức (3), (4) tính được toạ độ trắc đia $B, L$; theo công thức (1), (2) chuyển toạ độ $B, L$ về toạ độ $x, y$ tương ứng với kinh tuyến trục mới.

Khi chuyển đổi bản đồ số giữa các hệ toạ VN2000, WGS-84, chúng ta thực hiện theo các công thức (1),(2),(3),(4),(5),(6),(7) với trình tự sau:

$$
(x y h)_{v n} \text {---- }(B L H)_{v n} \text {---- }(X Y Z)_{v n} \text {---- }(X Y Z)_{\text {wgs }} \text {--- }(B L H)_{\text {wgs }} \text {--- }(x y h)_{\text {wgs }}
$$

Bằng ngôn ngữ lập trình VB 6.0 chúng ta thực hiện chuyển đổi tất cả các điểm. Kết quả cho độ tin cậy cao. Một số modul chuyển đổi cụ thể như sau: 


\section{Nghiên cúu - Úng dụng}

\begin{tabular}{|c|c|}
\hline $\begin{array}{l}\text { CHUYEN B L H SANG X Y Z } \\
\text { ro }=206264.806247096\end{array}$ & $\begin{array}{l}d p=\operatorname{sqr}(d x * d x+d y * d y) \\
\text { Bo }=\operatorname{atn}(d z *(1+e 12) / d p)\end{array}$ \\
\hline$a=6378137 \#$ & No $=a / \operatorname{sqr}\left(1-e 2{ }^{*} \sin (B o)^{\wedge} 2\right)$ \\
\hline e2 $=6.69438000426084 \mathrm{E}-03$ & $\mathrm{Bt}=\operatorname{atn}\left(\left(\mathrm{dz}+\mathrm{e} 2 * \mathrm{No}^{*} \sin (\mathrm{Bo})\right) / \mathrm{dp}\right)$ \\
\hline$N=a / \operatorname{sqr}\left(1-e 2{ }^{*} \sin (B) * \sin (B)\right)$ & $\mathrm{No}=\mathrm{a} / \operatorname{sqr}\left(1-\mathrm{e} 2{ }^{*} \sin (\mathrm{Bt})^{\wedge} 2\right)$ \\
\hline$X=(N+H){ }^{*} \cos (B) * \cos (L)$ & Eplon = abs(Bt - Bo) \\
\hline$Y=(N+H) * \cos (B) * \sin (L)$ & Do While Eplon > 1E-20 \\
\hline$Z=(N *(1 \#-e 2)+H) * \sin (B)$ & $\mathrm{Bo}=\operatorname{atn}\left(\left(\mathrm{dz}+\mathrm{e} 2{ }^{*} \mathrm{No}{ }^{*} \sin (\mathrm{Bt})\right) / \mathrm{dp}\right)$ \\
\hline CHUYEN XYZ SANG BLH & $\mathrm{No}=\mathrm{a} / \operatorname{sqr}\left(1-\mathrm{e} 2{ }^{*} \sin (\mathrm{Bo})^{\wedge} 2\right)$ \\
\hline $\mathrm{pi}=3.14159265358979$ & $\mathrm{Bt}=\operatorname{atn}\left(\left(\mathrm{dz}+\mathrm{e} 2{ }^{*} \mathrm{No}{ }^{*} \sin (\mathrm{Bo})\right) / \mathrm{dp}\right)$ \\
\hline$a=6378137 \#$ & $\mathrm{No}=\mathrm{a} / \operatorname{sqr}\left(1-\mathrm{e} 2{ }^{*} \sin (\mathrm{Bt})^{\wedge} 2\right)$ \\
\hline $\mathrm{e} 2=6.69438000426084 \mathrm{E}-03$ & Eplon $=$ abs $(\mathrm{Bt}-\mathrm{Bo})$ \\
\hline $\mathrm{e} 12=\mathrm{e} 2 /(1-\mathrm{e} 2)$ & Loop \\
\hline$d x=X$ & $\mathrm{~B}=\mathrm{Bt}$ \\
\hline$d y=Y$ & $H=d z / \sin (B)-N o *(1-e 2)$ \\
\hline$d z=Z$ & $\mathrm{~L}=\mathrm{pi}-\operatorname{atn}(\operatorname{abs}(\mathrm{dy} / \mathrm{dx}))$ \\
\hline
\end{tabular}

Private Sub dBLXY(ByVal a\#, e2\#, EP2\#, a0\#, A2\#, A4\#, A6\#, B\#, TL\#, tl2\#, x\#, y\#, tlk00\#, tl1\#)

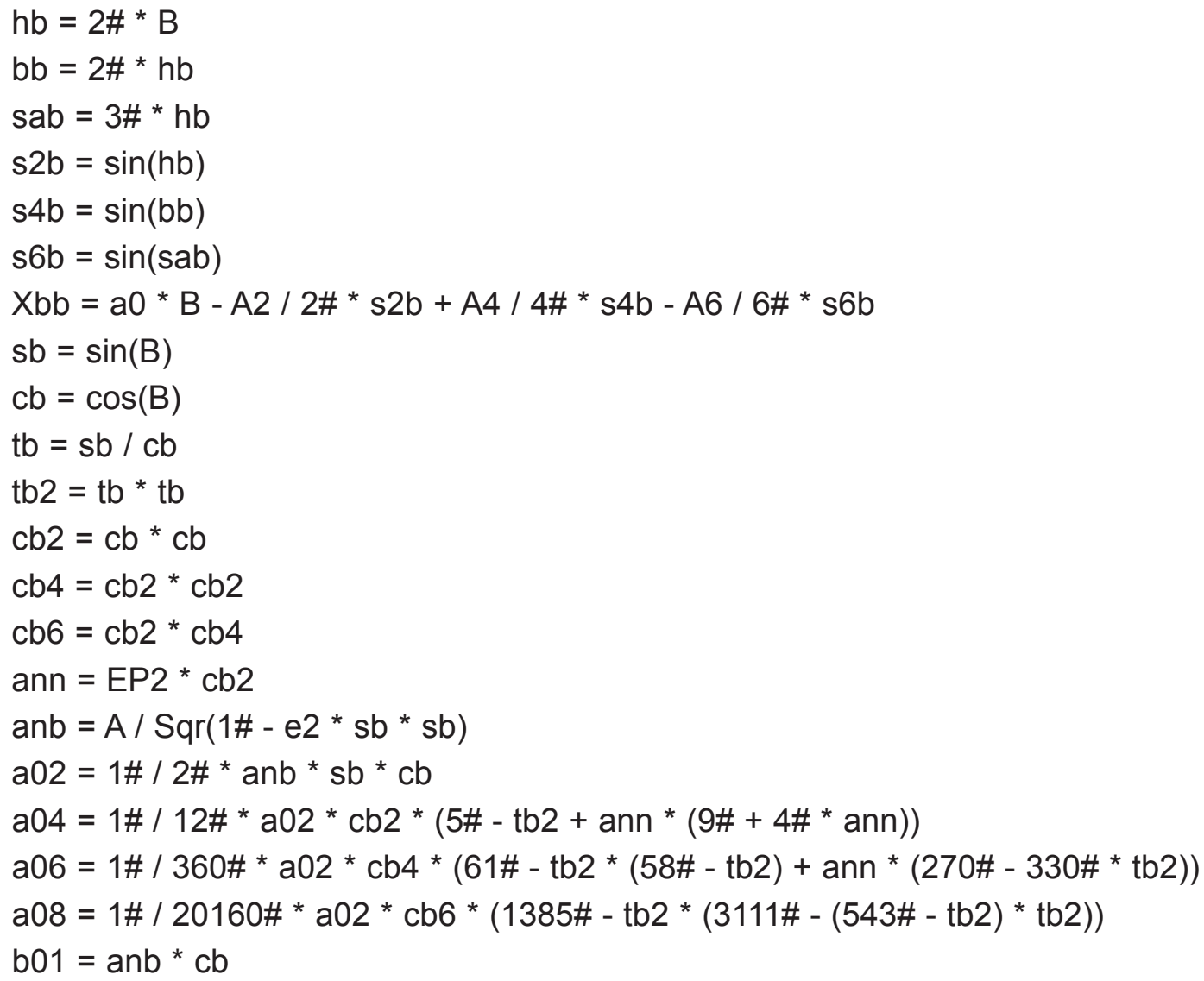




$$
\begin{aligned}
& \mathrm{b} 03=1 \# / 6 \#{ }^{*} \mathrm{~b} 01{ }^{*} \mathrm{cb} 2{ }^{*}(1 \#-\mathrm{tb} 2+\mathrm{ann}) \\
& \mathrm{b} 05=1 \# / 120 \#{ }^{*} \mathrm{~b} 01{ }^{*} \mathrm{cb} 4{ }^{*}\left(5 \#-\mathrm{tb} 2{ }^{*}(18 \#-\mathrm{tb} 2)+\mathrm{ann}{ }^{*}\left(14 \#-58 \#{ }^{*} \mathrm{tb} 2\right)\right) \\
& \mathrm{b} 07=1 \# / 5040 \#{ }^{*} \mathrm{~b} 01{ }^{*} \mathrm{cb} 6{ }^{*}\left(61 \#-\mathrm{tb} 2{ }^{*}\left(479 \#-\mathrm{tb} 2{ }^{*}(179 \#-\mathrm{tb} 2)\right)\right) \\
& \mathrm{al}=\mathrm{TL}-\mathrm{tl} 2 \\
& \mathrm{al} 2=\mathrm{al}{ }^{*} \mathrm{al} \\
& \mathrm{x}=\mathrm{tlk00}{ }^{*}\left(\mathrm{Xbb}+\left(\mathrm{a} 02+\left(\mathrm{a} 04+(\mathrm{a} 06+\mathrm{a} 08 * \mathrm{al} 2){ }^{*} \mathrm{al} 2\right){ }^{*} \mathrm{al} 2\right){ }^{*} \mathrm{al} 2\right) \\
& \mathrm{y}=500000 \#+\mathrm{tlk00}{ }^{*}\left(\left(\mathrm{~b} 01+\left(\mathrm{b} 03+\left(\mathrm{b} 05+\mathrm{b} 0{ }^{*} \mathrm{al} 2\right){ }^{*} \mathrm{al} 2\right){ }^{*} \mathrm{al} 2\right){ }^{*} \mathrm{al}\right)
\end{aligned}
$$

\section{End Sub}

Private Sub dXYBL(ByVal a\#, e2\#, EP2\#, a0\#, A2\#, A4\#, A6\#, Xo\#, Yo\#, TU\#, Xb\#, DK\#, tlk00\#, tl2\#)

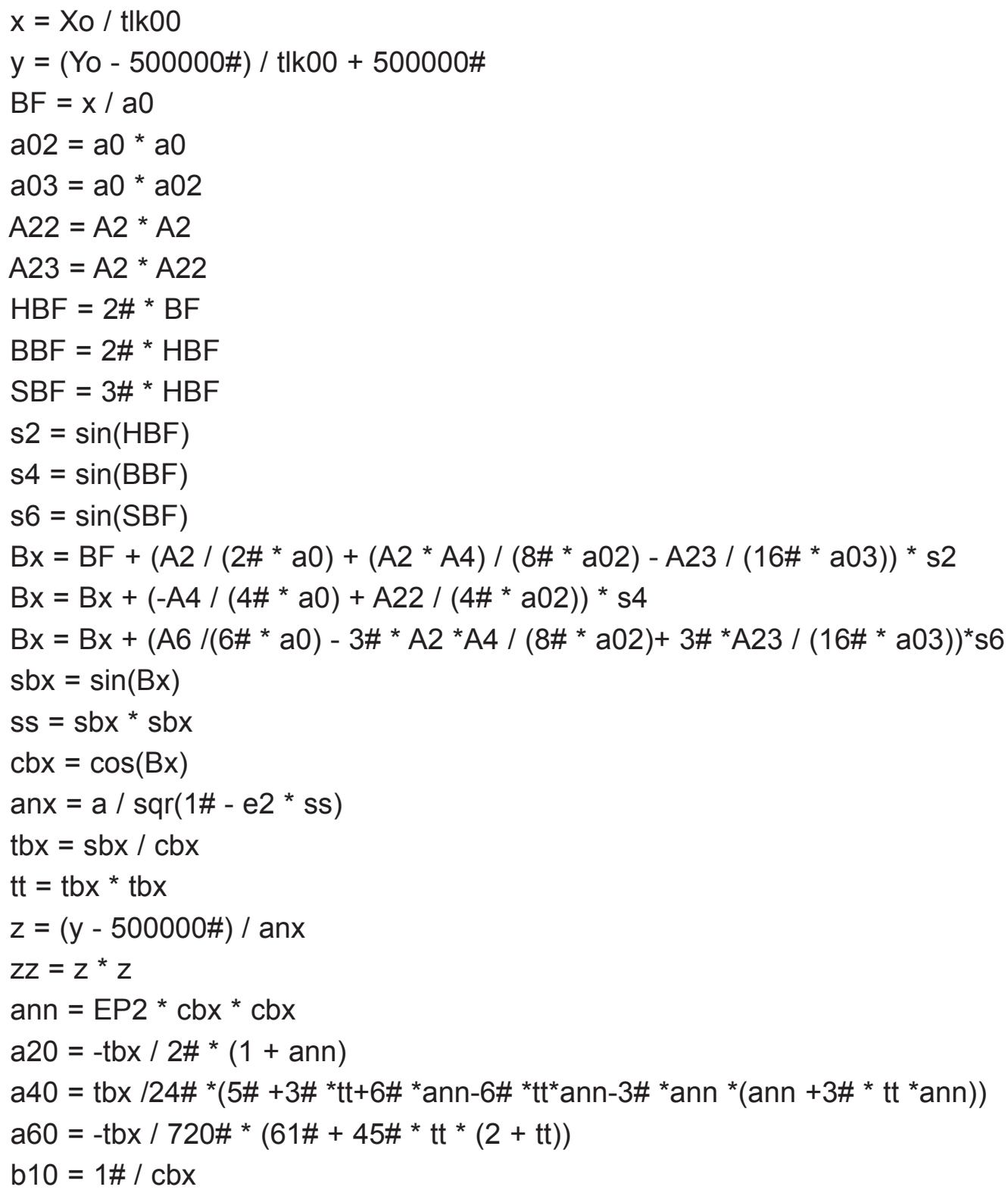




$$
\begin{aligned}
& \mathrm{b} 30=-\left(1 \#+2 \#{ }^{*} \mathrm{tt}+\mathrm{ann}\right) /\left(6 \#{ }^{*} \mathrm{cbx}\right) \\
& \mathrm{b} 50=\left(5 \#+\mathrm{tt}{ }^{*}\left(28 \#+24 \#{ }^{*} \mathrm{tt}\right)+\mathrm{ann}{ }^{*}\left(6 \#+8 \#{ }^{*} \mathrm{tt}\right)\right) /\left(120 \#{ }^{*} \mathrm{cbx}\right) \\
& X \mathrm{Xb}=\mathrm{Bx}+\left(\mathrm{a} 20+\left(\mathrm{a} 40+\mathrm{a} 60{ }^{*} \mathrm{zz}\right){ }^{*} \mathrm{zz}\right){ }^{*} \mathrm{zz} \\
& \mathrm{DK}=\mathrm{TU}+\left(\mathrm{b} 10+\left(\mathrm{b} 30+\mathrm{b} 50{ }^{*} \mathrm{zz}\right){ }^{*} \mathrm{zz}\right){ }^{*} \mathrm{z}
\end{aligned}
$$

\section{End Sub}

Trên cơ sở các modul chương trình, chúng ta có được kết quả chuyển đổi dữ liệu bản đồ số địa hình từ hệ WGS-84 quốc tế sang hệ VN2000 và ngược lại. Trong trường hợp bản đồ được thành lập ở các kinh tuyến trục khác nhau, kích thước múi khác nhau, chúng ta tiến hành chuyển đổi các điểm địa hình từ tọa độ $X, Y$ sang tọa độ $B, L$ rồi chuyển về tọa độ $X, Y$ của múi chiếu mới trong cùng một hệ thống tọa độ. Trong đó dữ liệu được nhận từ phần mềm đồ hoạ AutoCad, MicroStation, Mapinfo.

\section{Kết quả thực hiện} hinh)

a- Modul chuyển đổi bản đồ từ hệ WGS84 về VN2000 và VN2000 sang WGS84 (xem

b- Tọa độ các điểm địa hình khi thực hiện chuyển đổi hệ quy chiếu WGS84 và VN2000 (xem bảng)

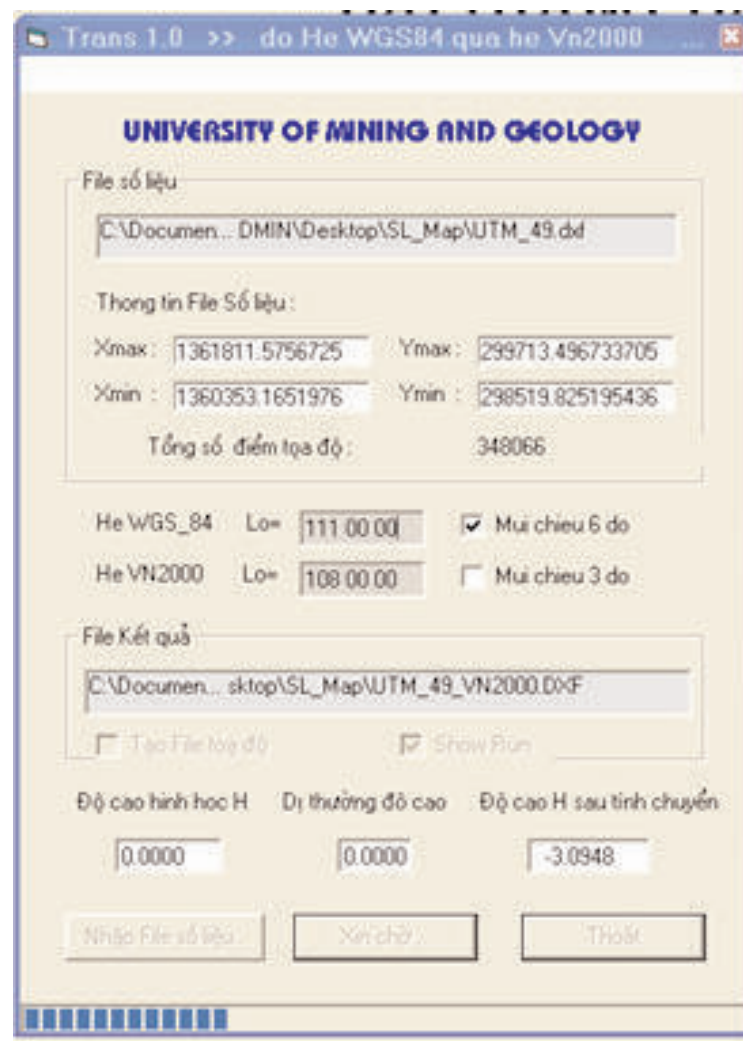

$5 / 22 / 2016$

Ts. Đinh Công Hòa

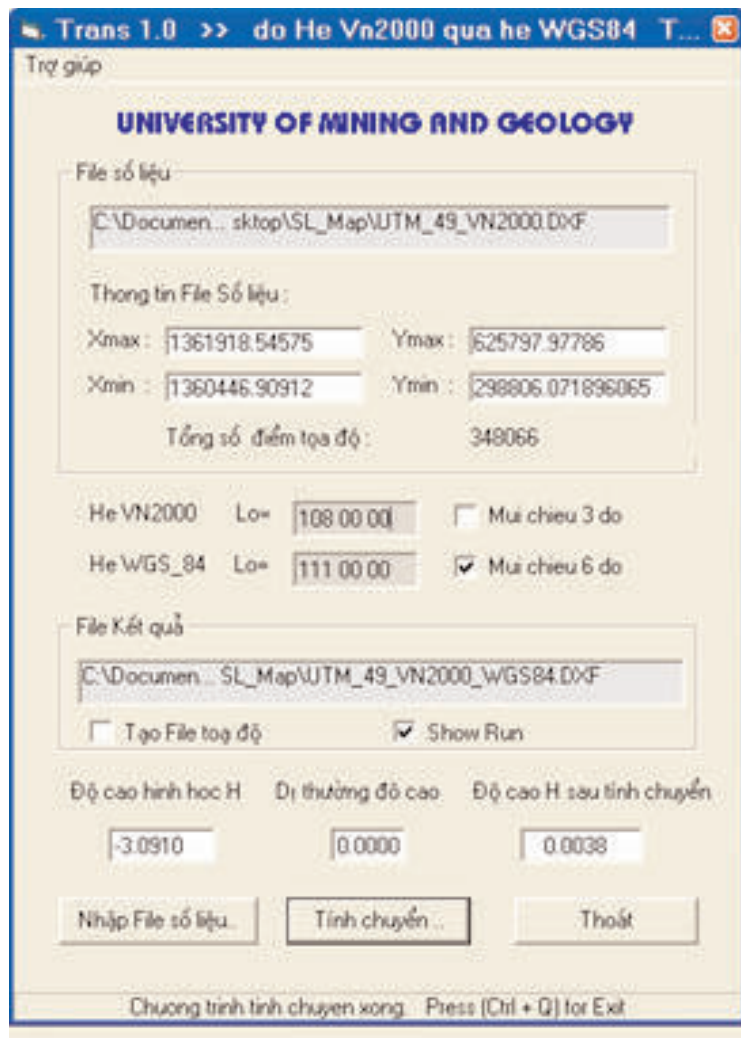

$5 / 23 / 2016$

Ts. Đinh Công Hòa 


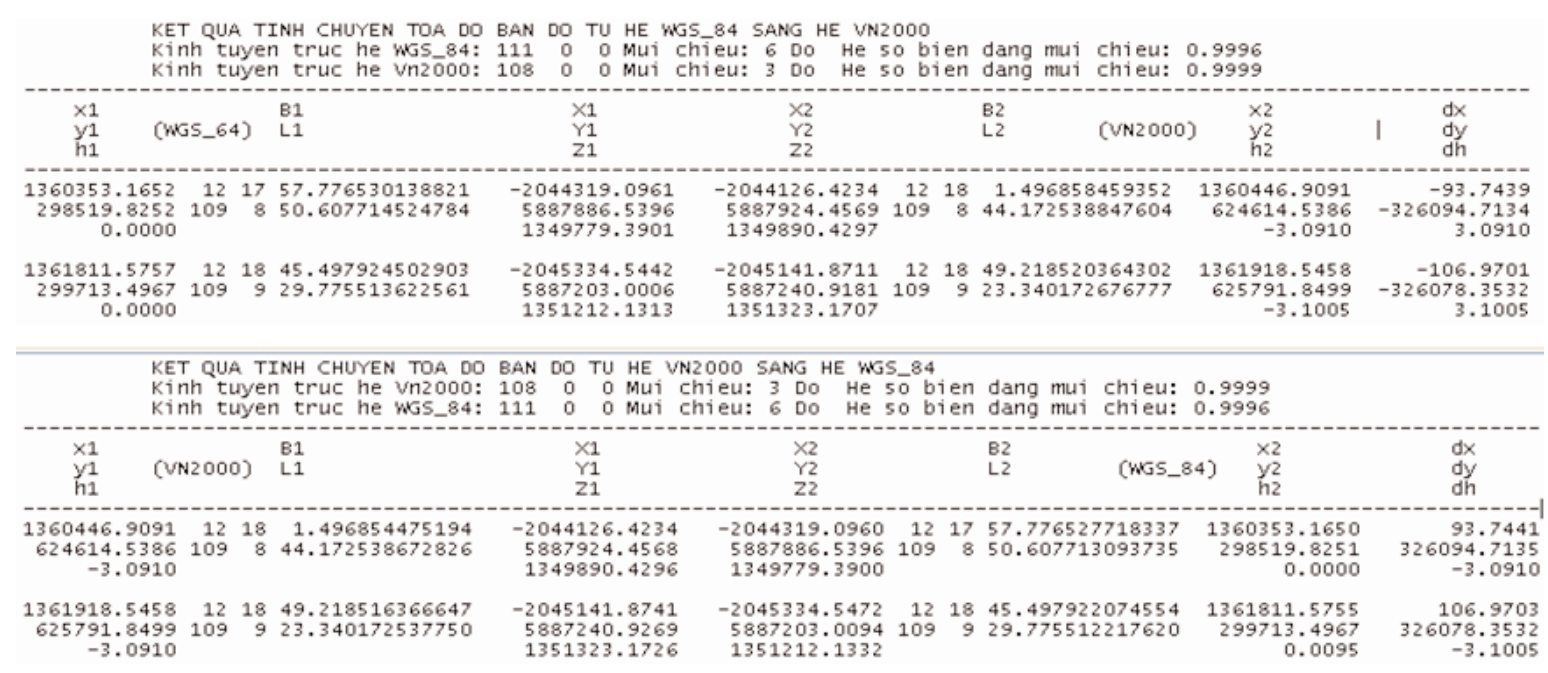

\section{Kết luận}

Để thực hiện đựơc những kết quả nêu trên, chúng tôi đã xây dựng các modul chương trình bằng ngôn ngữ lập trình Visual Basic 6.0, C++, các modul được kết nối với nhau tạo thành phần mềm ứng dụng tiện lợi và rễ dàng sử dụng. Những kết quả nghiên cứu đã được kiểm tra tính chính xác thông qua các dữ liệu chuẩn. Kết quả nghiên cứu đã được ứng dụng trong thực tế sản xuất. Bạn đọc có thể Download phần mềm Geosoft từ Website: www.dinhconghoa.com để được hiểu rõ hơn. $\bigcirc$

\section{Tài liệu tham khảo}

[1]. Đinh Công Hoà. 2011. Lập trình bài toán trắc địa cơ sở. NXB Giao thông Vận tải.

[2]. Thông tư 973/2001/TT-TCĐC hướng dẫn áp dụng hệ quy chiếu và hệ toạ độ quốc gia VN-2000 do Tổng cục Địa chính ban hành. $\mathrm{O}$

\section{Summary}

Apply information technology in problem of the transform reference system of digital topographic map

Dinh Cong Hoa, Hoang Thi Thuy

\section{Hanoi University of Mining and Geology}

Digital map can be established in coordinate systems with different central meridians in diversity of graphic data formats. Therefore, it is necessary to transform the maps into the same identified coordinate system for more effective management and application, especially in the era of international integration. To effectively perform this task, this study generated a software by linking various mudules programed by Visual Basic 6.0 and $\mathrm{C}++$. The program was tested on standard data. Recently, it has been beeing applied in real projects. $\bigcirc$ 